\title{
Cavity optomechanics with a trapped, interacting Bose-Einstein condensate
}

\author{
D. Nagy ${ }^{\mathrm{a}}$, G. Szirmai, and P. Domokos \\ Institute for Solid State Physics and Optics, Wigner Research Centre for Physics, Hungarian Academy of Sciences, P. O. Box \\ 49, H-1525 Budapest, Hungary
}

Received: date / Revised version: date

\begin{abstract}
The dispersive interaction of a Bose-Einstein condensate with a single mode of a high-finesse optical cavity realizes the radiation pressure coupling Hamiltonian. In this system the role of the mechanical oscillator is played by a single condensate excitation mode that is selected by the cavity mode function. We study the effect of atomic s-wave collisions and show that it merely renormalizes parameters of the usual optomechanical interaction. Moreover, we show that even in the case of strong harmonic confinementwhich invalidates the use of Bloch states - a single excitation mode of the Bose-Einstein condensate couples significantly to the light field, that is the simplified picture of a single "mechanical" oscillator mode remains valid.
\end{abstract}

PACS. 03.75.Kk Dynamic properties of condensates; collective and hydrodynamic excitations, superfluid flow $-37.10 . \mathrm{Vz}$ Mechanical effects of light on atoms, molecules, and ions - 37.30.+i Atoms, molecules, and ions in cavities

\section{Introduction}

Cavity optomechanics has seen a rapid expansion over recent years attracting great theoretical and experimental interest. The basic paradigm of coupling a vibrational mode of a mesoscopic object to an electromagnetic field mode via radiation pressure force has been realized in various systems (for a comprehensive review consult [1]). With nanomechanical oscillators, it still remains a challenge in the optical domain to reach simultaneously the quantum mechanical ground state and the quantum coherent regime, where the coupling exceeds both the optical and mechanical decoherence rates 2. In contrast, these goals are relatively easily achieved with ultracold atoms [3, 4, 5]. Most remarkably, radiation pressure coupling can be simulated with a Bose-Einstein condensate (BEC) dispersively coupled to the field of a high-finesse optical cavity, that can be either a ring cavity $[6,7,8,9]$ or a linear microcavity 10,11 . In the latter case, the cavity mode function selects a single excitation mode of the BEC, which plays the role of the mechanical oscillator. For a pure condensate the occupation of the selected excitation is already zero, thus one can readily study the quantum coherent regime of optomechanics. As compared to the single atom cavity QED, the coupling of the collective excitation mode to the field is enhanced by the square root of the atom number. In this strong coupling regime optical nonlinearity emerges on a single photon level [12, 13], which may give rise to photon blockade effect [14] and

\footnotetext{
a e-mail: nagy.david@wigner.mta.hu
}

non-Gaussian steady states 15. This regime also opens up the way to possible future applications of BEC-cavity systems as optical transistors [16] and switches [17].

Nonetheless, using a BEC excitation mode as a "mechanical" oscillator sets important limitations. The frequency of this matter-wave oscillator, defined by the recoil frequency $\omega_{R}$, is by orders of magnitudes lower than the coupling strength and usually than the cavity photon loss rate $\kappa$. As a result, the quantum noise associated with the photon leakage gives rise to significant incoherent excitations of the BEC. The rate of diffusion out of the ground state is comparable to the interaction strength, which leads to the dephasing of coherent oscillations 18 . On a longer time scale, the condensate depletes into the "mechanical" oscillator mode which approaches a steady state with average occupation number being in the order of $\kappa / \omega_{R}$ [19,20].

In this article, we shall study two further effects which are inherent to the BEC experimental approach to optomechanics and which might lead to significant departure from the realization of the radiation pressure coupling models. The first one is the effect of internal interactions on the collective excitation mode. Indeed, the effect of s-wave atom-atom collisions cannot be generally neglected, as it plays, e.g., a crucial role in the process of Bose-Einstein condensation, too. In a recent paper the collisional effects have been considered quite generally for the BEC-cavity system [21. Here we find for the special case of the simulated radiation pressure model that the internal interaction renormalizes the model parameters, 
however, the generic form remains good approximation. The second effect is due to the external trapping. Since the condensate is inhomogeneous and has a finite extent of a few wavelengths, the selection of a single "mechanical" mode is not perfectly warranted. We find that the single-mode approximation to optomechanics can be applied already for a trap size as small as 10 times the optical wavelength.

The paper is organized as follows: In Sec. 2 we introduce the dispersively coupled cavity and atom fields. Sec. 3 describes the cavity optomechanics with a homogeneous BEC. We develop a band model for the periodic system, and show that the cavity mode singles one excitation mode out of the continuum. We describe the effects of collisions on the dispersive bistability of the system both by a full mean-field model and by an effective radiation pressure coupling Hamiltonian. Sec. 4 investigates the limitations of optomechanics for a trapped BEC. Finally, we conclude in Sec. 5 .

\section{The BEC-cavity system}

We consider a zero-temperature Bose-Einstein condensate trapped inside a high-finesse optical resonator. The atoms interact with a single cavity mode having frequency $\omega_{C}$ and mode function $\cos k x$, where $k=2 \pi / \lambda$ is the wave number. The system is driven through one of the cavity mirrors by a coherent laser field of frequency $\omega$ which is far detuned from the atomic transition $\omega_{A}$, i.e., the atomic detuning $\Delta_{A}=\omega-\omega_{A}$ far exceeds the atomic linewidth $\gamma$, hence spontaneous emission is suppressed. Meanwhile, the laser field is nearly resonant to the cavity mode, $\left|\Delta_{C}\right|=\omega-\omega_{C} \approx \kappa$, with $2 \kappa$ being the cavity mode linewidth. We assume a strong dipole coupling between the atoms and the mode characterized by the singlephoton Rabi frequency $\Omega$ which is in the order of the dissipation rates $\kappa, \gamma$. In this limit the strength of the dispersive atom-field interaction is defined by the one-atom light shift $U_{0}=\frac{\Omega^{2}}{\Delta_{A}}[22$. In the frame rotating with the laser frequency $\omega$, the many-particle Hamiltonian reads [20]

$$
\begin{aligned}
H / \hbar= & -\Delta_{C} a^{\dagger} a+\eta\left(a+a^{\dagger}\right) \\
& +\int \Psi^{\dagger}(x)\left[-\frac{\hbar}{2 m} \frac{d^{2}}{d x^{2}}+V_{\text {ext }}(x)\right. \\
& \left.+\frac{g}{2} \Psi^{\dagger}(x) \Psi(x)+U_{0} a^{\dagger} a \cos ^{2}(k x)\right] \Psi(x) d x,
\end{aligned}
$$

where $\Psi(x)$ is the atom field operator and $a$ is the cavity mode operator. We consider the dynamics in one dimension $x$ along the cavity axis. The first two terms describe the radiation field and the pumping of the cavity mode. The energy scale of the atomic motion is given by the recoil frequency $\omega_{R}=\hbar k^{2} /(2 m)$. The external trapping potential $V_{\text {ext }}(x)$ is assumed to vary slowly on the wavelength scale. We include s-wave atom-atom collisions with the $1 \mathrm{D}$ interaction parameter $g$, which is proportional to the s-wave scattering length. The last term accounts for the dispersive light-matter interaction between the atoms and the mode.

In cavity optomechanics one has to consider a dissipative dynamics for the photon field due to the photon leakage through the cavity mirrors. We take this into account in the Heisenberg-Langevin equations of motion of the field operator $[19,20]$

$$
\frac{d}{d t} \hat{a}=-\frac{i}{\hbar}[\hat{a}, H]-\kappa \hat{a}+\hat{\xi},
$$

where $2 \kappa$ is the photon loss rate and the operator $\xi$ describes the measurement back-action noise with the only non-zero correlation function $\left\langle\hat{\xi}(t) \hat{\xi}^{\dagger}\left(t^{\prime}\right)\right\rangle=\kappa \delta\left(t-t^{\prime}\right)$.

\section{Optomechanical coupling in a band model}

The system can be considerably simplified when the condensate is homogeneous. In this case, the cavity field couples exclusively to a single excitation mode of the BEC, thus an effective radiation pressure Hamiltonian can be constructed for these two modes. The effect of atom-atom collisions is that they renormalize the optomechanical coupling strength and the frequency of the "mechanical" mode.

In the absence of an external potential, $V_{\text {ext }}(x) \equiv 0$, the problem is periodic along the cavity axis. The interaction term in Hamiltonian (1) has the periodicity of $\lambda / 2$, where $\lambda$ is the optical wavelength. It follows that one can introduce a band model [20,23] by expanding the atomic field operators in terms of Bloch functions,

$$
\begin{aligned}
& \Psi(x)=\frac{1}{\sqrt{L}} \sum_{q} e^{i q x} \\
& \left\{b_{q}+\sqrt{2} \sum_{n}\left[c_{n, q} \cos 2 n k x+s_{n, q} \sin 2 n k x\right]\right\},
\end{aligned}
$$

where $n$ is the band index and $q \in[-k, k]$ denotes the quasimomentum in the first Brillouin zone, and $L$ is the linear size of the system. $b_{q}, c_{n, q}$ and $s_{n, q}$ are annihilation operators of the corresponding states. We use the standing wave basis $e^{i q x} \cos 2 n k x$, and $e^{i q x} \sin 2 n k x$ instead of plane waves because the atom-field interaction directly populates the cosine wave functions with $q=0$.

The energy of the bands depend quadratically on the band index, $E_{n, q=0}=(2 n)^{2} \omega_{R}$, hence there is a hierarchy in their population as shown in Ref. 24. We truncate the expansion Eq. (3) at $n=1$, meaning that we make a three-band approximation and restrict the dynamics in the three lowest lying bands $b_{q}, c_{1, q} \rightarrow c_{q}$ and $s_{1, q} \rightarrow s_{q}$. Accordingly,

$$
\int_{0}^{L} \Psi^{\dagger}(x) \Psi(x) d x=\sum_{q}\left[b_{q}^{\dagger} b_{q}+c_{q}^{\dagger} c_{q}+s_{q}^{\dagger} s_{q}\right]=N .
$$

This is valid when the optical potential depth $U_{0}\left\langle a^{\dagger} a\right\rangle$ is below the recoil frequency. 
By inserting Eq. (3) into Hamiltonian (1) (but keeping only $n=1$ from the sum), we obtain the following secondquantized form,

$$
\begin{array}{r}
H=-\delta_{C} a^{\dagger} a+\eta\left(a+a^{\dagger}\right)+\frac{U_{0}}{2 \sqrt{2}} a^{\dagger} a \sum_{q}\left(b_{q}^{\dagger} c_{q}+c_{q}^{\dagger} b_{q}\right) \\
+H_{\text {kin }}+H_{\text {coll }},
\end{array}
$$

where we introduced the shifted cavity detuning $\delta_{C}=$ $\Delta_{C}-\frac{N U_{0}}{2}$. The atom-cavity interaction (third term) couples the $b_{q}$ modes to the $c_{q}$ modes preserving the quasi momentum $q$. The kinetic energy reads

$$
\begin{aligned}
H_{\mathrm{kin}}=\omega_{R} \sum_{q}\left[q^{2} b_{q}^{\dagger} b_{q}+\left(q^{2}+4\right)\left(c_{q}^{\dagger} c_{q}+s_{q}^{\dagger} s_{q}\right)\right. \\
\left.+4 i q\left(c_{q}^{\dagger} s_{q}-s_{q}^{\dagger} c_{q}\right)\right] .
\end{aligned}
$$

Note that the sine modes $s_{q}$, not present in the atomcavity coupling (third term in Eq. (5)), mix with the cosine modes $b_{q}$ here. They are thus needed to recover the correct excitation spectrum. Finally, the collisions are represented by

$$
\begin{gathered}
H_{\text {coll }}=\frac{g}{2} \frac{\lambda}{L} \sum_{q_{1}, q_{2}, q_{3}, q_{4}} \delta_{q_{1}+q_{2}-q_{3}-q_{4}}[ \\
b_{q_{1}}^{\dagger} b_{q_{2}}^{\dagger} b_{q_{3}} b_{q_{4}}+\frac{3}{2} c_{q_{1}}^{\dagger} c_{q_{2}}^{\dagger} c_{q_{3}} c_{q_{4}}+\frac{3}{2} s_{q_{1}}^{\dagger} s_{q_{2}}^{\dagger} s_{q_{3}} s_{q_{4}} \\
+b_{q_{1}}^{\dagger} b_{q_{2}}^{\dagger} c_{q_{3}} c_{q_{4}}+b_{q_{1}}^{\dagger} b_{q_{2}}^{\dagger} s_{q_{3}} s_{q_{4}}+\frac{1}{2} c_{q_{1}}^{\dagger} c_{q_{2}}^{\dagger} s_{q_{3}} s_{q_{4}}+\text { h.a. } \\
\left.+4 b_{q_{1}}^{\dagger} c_{q_{2}}^{\dagger} b_{q_{3}} c_{q_{4}}+4 b_{q_{1}}^{\dagger} s_{q_{2}}^{\dagger} b_{q_{3}} s_{q_{4}}+2 c_{q_{1}}^{\dagger} s_{q_{2}}^{\dagger} c_{q_{3}} s_{q_{4}}+\text { h.a. }\right]
\end{gathered}
$$

The s-wave atom-atom scattering mixes operators with different $n$ and $q$ preserving the total momentum. In the above Hamiltonian, we neglected Umklapp scattering processes, where the band momentum is transformed into quasi momentum, and considered only normal scattering where the quasi momentum is preserved, i.e. $q_{1}+q_{2}-q_{3}-$ $q_{4}=0$.

\subsection{Mean-field description}

In order to describe the coupled dynamics of the BEC and the photon field we employ the same technique [20] that leads to the Gross-Pitaevskii equation for a Bose gas. Hence, the operators are split into their mean values and fluctuations,

$$
\begin{aligned}
a(t) & =\alpha+\delta a, \\
b_{q}(t) & =e^{-i \mu t}\left[\sqrt{N} \beta_{q}+\delta b_{q}\right], \\
c_{q}(t) & =e^{-i \mu t}\left[\sqrt{N} \gamma_{q}+\delta c_{q}\right], \\
s_{q}(t) & =e^{-i \mu t}\left[\sqrt{N} \sigma_{q}+\delta s_{q}\right] .
\end{aligned}
$$

For the atoms the mean field can be interpreted as the wave function of the condensate with components $\beta_{q}, \gamma_{q}$ and $\sigma_{q}$ in the corresponding states, and chemical potential $\mu$. Note that the wave function can be chosen real. Momentum is conserved simultaneously for the mean field and for the fluctuations. If initially a homogeneous condensate is prepared in the zero momentum state with only $\beta_{0} \neq 0$, then due to momentum conservation the condensate wave function stays in the $q=0$ subspace even after adiabatically turning on the cavity field. Furthermore the parity is conserved, therefore the atom-cavity interaction populates only $\gamma_{0}$, and leaves $\sigma_{0}=0$.

One obtains the following three coupled equations in the steady state for the mean fields by taking the average of the Heisenberg-Langevin equation of motion [Eq. (2) and similarly for the atomic fields from the Hamiltonian (5)]:

$$
\begin{gathered}
\alpha=\frac{\eta}{i \kappa+\left(\delta_{C}-2 N u \beta_{0} \gamma_{0}\right)}, \\
\mu \beta_{0}=u|\alpha|^{2} \gamma_{0}+\mathcal{G}\left(\beta_{0}^{3}+3 \beta_{0} \gamma_{0}^{2}\right), \\
\mu \gamma_{0}=4 \omega_{R} \gamma_{0}+u|\alpha|^{2} \beta_{0}+\mathcal{G}\left(\frac{3}{2} \gamma_{0}^{3}+3 \beta_{0}^{2} \gamma_{0}\right),
\end{gathered}
$$

together with the normalization $\beta_{0}^{2}+\gamma_{0}^{2}=1$. For brevity, we introduced the notations $u=\frac{U_{0}}{2 \sqrt{2}}$ and the collisional parameter $\mathcal{G}=g N \lambda / L$.

The equation of motion of the fluctuations are linearized around the mean field solution. As a result of momentum conservation the equations decouple for distinct quasimomenta. S-wave scattering mixes the $\pm q$ quasimomentum states, hence, for each $q \neq 0$, one has to solve six coupled equations. This is precisely the Bogoliubov problem for a BEC in a periodic potential (restricted to the lowest three bands). By arranging the variables into $\underline{R}_{q}=\left[\delta b_{q}, \delta b_{-q}^{\dagger}, \delta c_{q}, \delta c_{-q}^{\dagger}, \delta s_{q}, \delta s_{-q}^{\dagger}\right]^{T}$, the following compact notation is obtained for $q \neq 0$ :

$$
i \frac{d}{d t} \underline{R}_{q}=\mathbf{L}_{q} \underline{R}_{q}
$$

where $\mathbf{L}_{q}$ is a six by six matrix whose elements depend on the mean-field solution and the quasi momentum $q$ (for the complete form of $\mathbf{L}_{q}$ see the Appendix). The $q=0$ fluctuations have to be treated differently. Within the mean-field model, the cavity field fluctuations $(\delta a)$ couple only to the even parity states with $q=0\left(\delta b_{0}\right.$ and $\left.\delta c_{0}\right)$. These operators can be arranged accordingly into the vector $\underline{R}=\left[\delta a, \delta a^{\dagger}, \delta b_{0}, \delta b_{0}^{\dagger}, \delta c_{0}, \delta c_{0}^{\dagger}\right]^{T}$, and the coupled BEC-cavity excitations are described by

$$
i \frac{d}{d t} \underline{R}=\mathbf{M} \underline{R}+\underline{\xi}
$$

with $\mathbf{M}$ being a six by six complex matrix that depends on the mean field solution (for its complete form see the Appendix). The cavity field fluctuations are driven by the dissipation noise that is arranged into the vector $\underline{\xi}=$ $\left[\xi, \xi^{\dagger}, 0,0,0,0\right]^{T}$. 


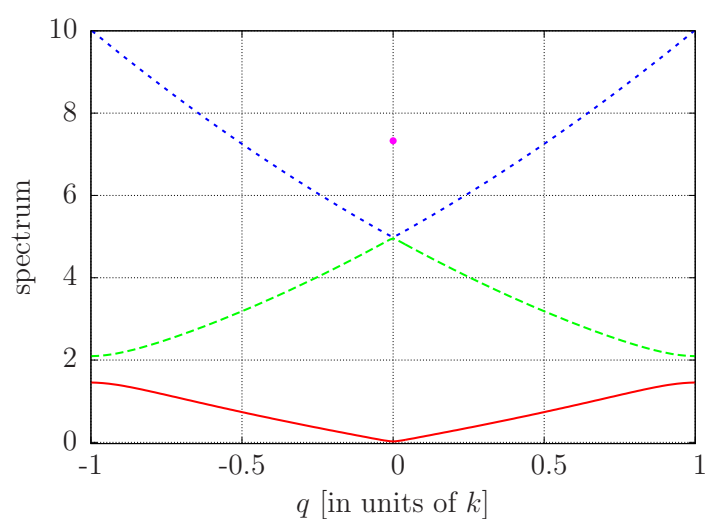

Fig. 1. Band structure of the BEC excitation spectrum. The energy eigenstates for $q \neq 0$ depends on the cavity field only through the mean photon number. The optical potential opens gaps between adjacent bands. A single $q=0$ state (filled circle) is pulled out from the third band (dashed blue) by the interaction with cavity field fluctuations. The parameters are $N=6 \cdot 10^{4}, U_{0}=0.96, \eta=549.5, \kappa=363.9, \delta_{C}=-5120$ and $\mathcal{G}=1 \omega_{R}$.

The excitation spectrum of the BEC interacting with the cavity field is depicted in Fig. 1. The periodic optical potential leads to a band structure, as it opens gaps at the center and at the edges of the Brillouin zone. In the first band, the spectrum starts linearly in $q$, that is typical to the Bogoliubov spectrum of a homogeneous BEC. Interaction with the photon field fluctuations picks out a single mode with $q=0$ from the upper band, which constitutes the optomechanical mode. In the following we will concentrate on this relevant mode, and derive an effective radiation pressure Hamiltonian that includes s-wave collisions.

\subsection{Radiation pressure Hamiltonian}

Radiation pressure coupling between the cavity mode $a$ and the single BEC excitation mode $c_{0}$ can be obtained from Hamiltonian (5) by restricting the dynamics in the subspace of the modes $b_{0}$ and $c_{0}$.

The Hamiltonian in the restricted subspace (up to second order in $c_{0}$ and $c_{0}^{\dagger}$ ) reads

$$
\begin{aligned}
& H=-\delta_{C} a^{\dagger} a+4 \omega_{R} c_{0}^{\dagger} c_{0}+u a^{\dagger} a\left(b_{0}^{\dagger} c_{0}+c_{0}^{\dagger} b_{0}\right) \\
& +\frac{g}{2} \frac{\lambda}{L}\left(b_{0}^{\dagger} b_{0}^{\dagger} c_{0} c_{0}+c_{0}^{\dagger} c_{0}^{\dagger} b_{0} b_{0}+4 b_{0}^{\dagger} c_{0}^{\dagger} b_{0} c_{0}+b_{0}^{\dagger} b_{0}^{\dagger} b_{0} b_{0}\right) .
\end{aligned}
$$

First let us exploit the relation $b_{0}^{\dagger} b_{0}=N-c_{0}^{\dagger} c_{0}$ (Eq. (4)) in the last term of Eq. (12), and then apply the next level of approximation: $b_{0} \equiv \sqrt{N}$. By this we assume that the condensate is mainly homogeneous, and the relevant dynamics takes place in the $c_{0}$ mode. This approximation is valid in the limit $u|\alpha|^{2} \ll 4 \omega_{R}$. Keeping only the quadratic terms from the s-wave collisions, one ends up with the op- tomechanical Hamiltonian up to second order in the operators,

$$
H=-\delta_{C} a^{\dagger} a+\frac{\omega_{M}}{2}\left(X^{2}+Y^{2}\right)+G a^{\dagger} a X+\mathcal{G} X^{2},
$$

where we introduced the quadratures $X=\left(c_{0}^{\dagger}+c_{0}\right) / \sqrt{2}$, $Y=\left(c_{0}^{\dagger}+c_{0}\right) / \sqrt{2}$. The "mechanical" frequency is $\omega_{M}=$ $4 \omega_{R}$ and the coupling constant is $G=\sqrt{2 N} u$. The s-wave interaction acts on the $X$ quadrature in the last term, that can be eliminated by the Bogoliubov transformation $\widetilde{X}=\chi X$ and $\widetilde{Y}=Y / \chi$. With the new operators, we obtain the usual optomechanical Hamiltonian

$$
H=-\delta_{C} a^{\dagger} a+\frac{\widetilde{\omega}_{M}}{2}\left(\widetilde{X}^{2}+\widetilde{Y}^{2}\right)+\widetilde{G} a^{\dagger} a \widetilde{X},
$$

where the transformation parameter $\chi$ depends on the strength of the s-wave interaction as $\chi=\sqrt[4]{\left(\omega_{M}+2 \mathcal{G}\right) / \omega_{M}}$, and it rescales both the mechanical frequency

$$
\widetilde{\omega}_{M}=\sqrt{\omega_{M}\left(\omega_{M}+2 \mathcal{G}\right)},
$$

and the coupling constant

$$
\widetilde{G}=G / \chi
$$

This is one of the main results of the present paper: the presence of atom-atom collisions besides shifting the oscillator frequency also renormalizes the optomechanical coupling. In the regime of weak collisions, where $\mathcal{G} \ll \omega$, one obtains from the Taylor series a linear frequency shift $\widetilde{\omega}=\omega+\mathcal{G}$ and a coupling constant $\widetilde{G}=(1-\mathcal{G} /(2 \omega)) G$. The s-wave collision between the atoms increases thus the mechanical frequency and decreases the coupling strength.

\subsection{Effects of collisions on the dispersive bistability}

The nonlinear coupling between the BEC and the cavity mode results in a bistable behavior of the system, that is one has two stable mean-field solutions corresponding to different photon numbers and oscillator displacements. This is a key feature of radiation pressure coupling. In the following we compare the mean-field model, Eqs. (9a-c) to the mean-field solution of the effective optomechanical model. This latter one is obtained from the steady-state solution of Eq. (14) and Eq. (2) for the mean fields. By expressing $\langle X\rangle$, one obtains the following cubic equation for the mean cavity photon number $I=|\alpha|^{2}$,

$$
\frac{\widetilde{G}^{4}}{\widetilde{\omega}^{2}} I^{3}+2 \delta_{C} \frac{\widetilde{G}^{2}}{\widetilde{\omega}} I^{2}+\left(\delta_{C}^{2}+\kappa^{2}\right) I-\eta^{2}=0,
$$

Generally, this equation has one real and two complex solutions. However above a bistability threshold $\eta^{2} \geq \frac{8}{3 \sqrt{3}} \frac{\widetilde{\omega} \kappa^{3}}{\widetilde{G}^{2}}$, there is a finite interval in $\delta_{C}$ for which it has three real solutions. One of them is unstable and the other two are stable, which amounts to optical bistability. In the top panel of Fig. 2 we plot the solutions of Eq. (16) (with 

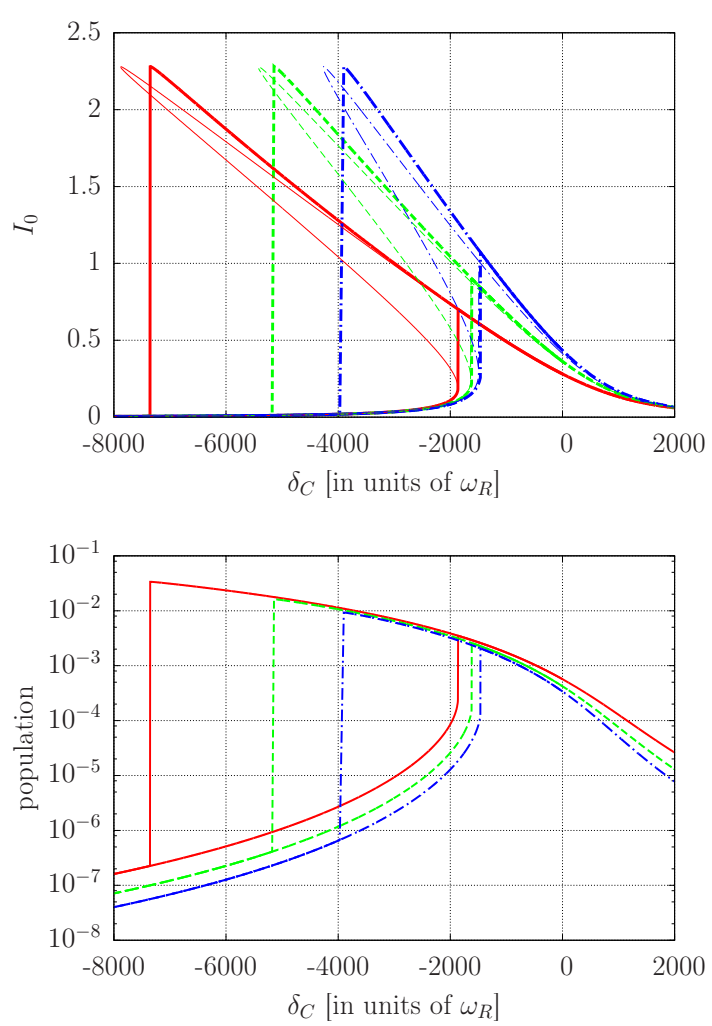

Fig. 2. Effects of collisions on bistability. Intracavity photon number $I_{0}$ (top panel) and the population of the optomechanical mode $\left|\gamma_{0}\right|^{2}$ (bottom panel) vs the detuning parameter $\delta_{C}$. From the collisionless case (solid red line), the s-wave collision parameter is increased to $\mathcal{G}=1$ (dashed green line) and $\mathcal{G}=2$ (dashed dotted blue line). On the top panel the thick lines represent the solutions of Eqs. (9a-c) while the thin lines show all of the three solutions of Eq. (16). The parameters reflects the experimental situation of Ref. [1], $N=6 \cdot 10^{4}, U_{0}=0.96$ $\eta=549.5, \kappa=363.9 \omega_{R}$.

thin lines) and compare them to the solutions of the full mean-field model Eqs. (9a-c) (thick lines) for three different collision parameters $\mathcal{G}=0$ (solid red), $\mathcal{G}=1$ (dashed green) and $\mathcal{G}=2$ (dashed dotted blue). As $\mathcal{G}$ increases, the width of the bistable region decreases, while its boundaries move upward in $\delta_{C}$. It is clearly seen that for photon numbers higher than 1 , the optomechanical model gets less exact quantitatively. The underlying reason is that this model discards the depletion of the mode $b_{0}$. The bottom panel of Fig. 2 shows the mean-field occupation of the optomechanical mode $\left|\gamma_{0}\right|^{2}$. One can observe that the s-wave interaction reduces the population $\left|\gamma_{0}\right|^{2}$, thus smearing out the density modulation above the homogeneous atom cloud. The decreasing $\left|\gamma_{0}\right|^{2}$ pulls the cavity closer to resonance (cf. Fig 2). Correspondingly, the photon number increases with $\mathcal{G}$ at a given $\delta_{C}$ on the upper branch.

In this section we focus on the behavior of the optomechanical mode (represented by the filled circle in Figure 1) across the bistable regime. Its frequency is significantly modulated by the interaction with the cavity field as shown in Fig. 3 (top panel). Far from the resonance,
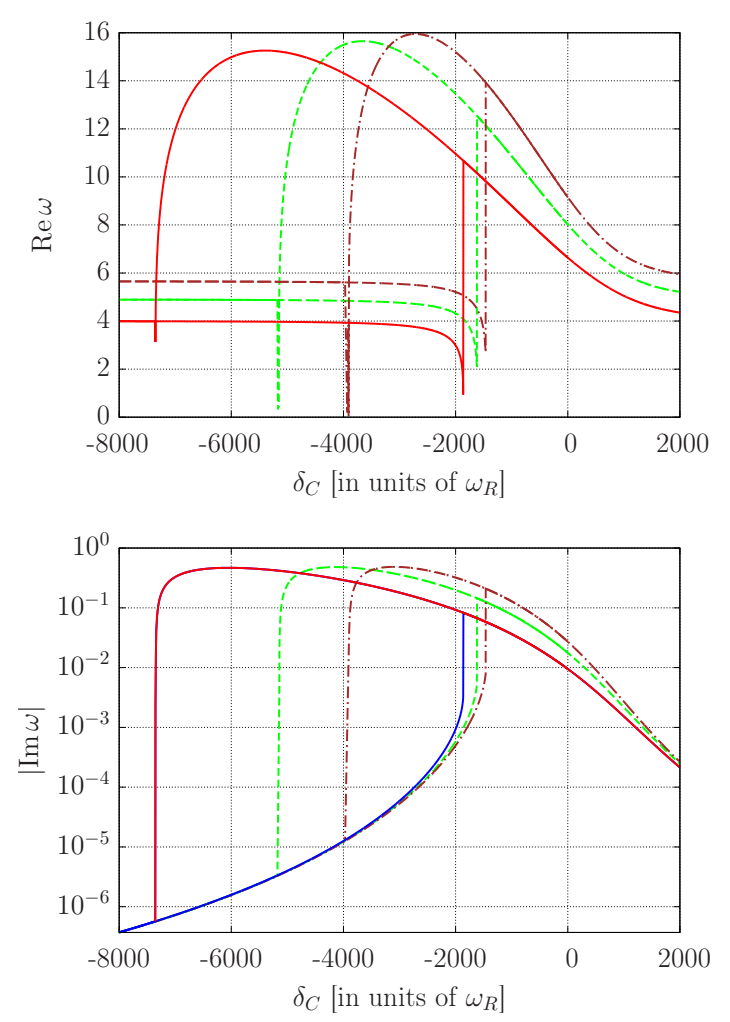

Fig. 3. Real part (top) and the absolute value of the imaginary part (bottom) of the optomechanical mode frequency as a function of the cavity detuning for $\mathcal{G}=0$ (solid red and blue lines), 1 (dashed green line) and 2 (dashed dotted brown line). Other parameters are the same as in Fig. 2

the frequency tends to $4 \omega_{R}$ for $\mathcal{G}=0$ (solid red line), and to higher values given by Eq. 1 a for $\mathcal{G} \neq 0$. In the bistability regime obtained at the vicinity of the cavity resonance, the optomechanical mode frequency is decreased by the BEC-cavity interaction on the lower branch, while it is increased on the upper branch. At the boundaries of the bistable regime, the frequency that corresponds to the vanishing branch sharply drops down to zero. The dissipative nature of the cavity mode gives rise to cooling ( $\operatorname{Im} \omega<0$, lower branch) or heating ( $\operatorname{Im} \omega>0$, upper branch) of the optomechanical mode [20]. On the bottom panel of Fig. 3 we plot the absolute value of the imaginary part of the complex frequency in logarithmic scale. (For $\mathcal{G}=0$ (solid line) we indicate cavity cooling with blue, and cavity heating with red colors.)

\section{Trapped condensate}

So far we have seen that a single excitation mode is sufficient to seize most of the physics of a BEC-cavity optomechanical system. Now we turn to the central issue of this article, that is, under what conditions the single-mode effective optomechanical model can be valid for a trapped atom gas. In the experiments [10,11, the condensate is kept inside the cavity mode volume with an extra dipole trap that breaks the discrete translational symmetry of 


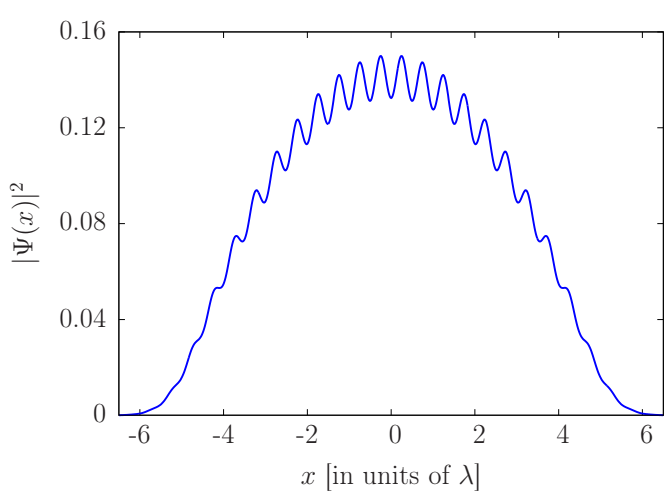

Fig. 4. The BEC wave function for $N=6 \cdot 10^{4}, U_{0}=0.96$, $\eta=549.5, \delta_{C}=0, g N=2, V_{t r}=0.01 \omega_{R}$. The corresponding Thomas-Fermi radius is $5.31 \lambda$.

the condensate. The quasi momentum $q$ is no longer a good quantum number. The dimension of the BEC along the cavity axis is typically around $10 \mu \mathrm{m}$, which is around 10-15 wavelengths. As a result, the selection of the optomechanical mode by the cavity mode is not perfect, and there can be more BEC excitation modes that couple to the cavity field. Moreover, atom-atom collision becomes significant too in shaping the condensate. Therefore the Bloch state expansion of the BEC excitations is not suitable any more. In the following, we investigate what are the relevant optomechanical modes and how they are composed of the discrete condensate excitation modes of a trapped BEC.

We consider Eqs. (1) and (2) in real space using a mean-field model similar to the one described in Sec. 3.1 We assume an external harmonic trap potential for the atoms, $V_{\text {ext }}(x)=V_{t r} x^{2}$. Similarly to the procedure of Sec. 3.1, we split the atomic field to a condensate meanfield wave function $\psi(x)$ and fluctuations $\delta \Psi(x, t)$,

$$
\Psi(x, t)=\sqrt{N} e^{-i \mu t}[\psi(x)+\delta \Psi(x, t)] .
$$

We solve the mean field equations (Eqs. (4a-b) of Ref. 20]) for a condensate size of 6 to $10 \lambda$ by discretizing the coordinate on 800 to 1400 grid points. A typical wave function is shown in Fig. 4 . For the selected parameters, the condensate takes the shape of the parabolic trap, i.e., it is deeply in the Thomas-Fermi regime. However, it is slightly modulated by the cavity field potential. This modulated component of the wave function corresponds to the $\cos 2 k x$ mode in the homogeneous case in Eq. (3). Here, the wave function can be approximated by $\psi(x)=$ $e(x)+\sqrt{2} \cos (2 k x) f(x)$, where $e(x)$ and $f(x)$ are slowly varying functions on the scale of $\lambda$.

Obviously, the system exhibits similar bistable behavior as in the untrapped case. In Fig. 5 we plot the cavity photon number as a function of the cavity detuning $\delta_{C}$ for fixed $g N=2$ but for two different trap frequencies $V_{t r}$. Note that for a trapped BEC, the s-wave interaction energy depends on the size of the condensate. In contrary to the homogeneous case, the parabolic trapping potential introduces a length scale and controls the condensate density and ultimately the s-wave scattering. When $V_{t r} \rightarrow 0$

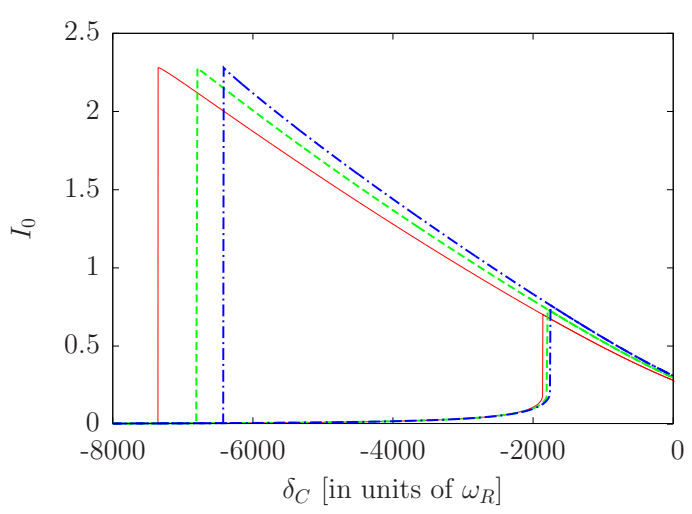

Fig. 5. The intracavity photon number $I_{0}$ vs the detuning parameter $\delta_{C}$ for a trapped BEC with Thomas-Fermi radii 3.11 $\lambda\left(V_{t r}=0.05\right)$ (blue dashed dotted line) and $5.31 \lambda\left(V_{t r}=0.01\right)$ (green dashed line). The parameters are: $N=6 \cdot 10^{4}, U_{0}=0.96$, $\eta=549.5, \kappa=363.9 \omega_{R}$ and $g N=2$. The noninteracting limit $(g N=0)$ of the homogeneous condensate $\left(V_{t r}=0\right)$ is plotted for reference (solid red line).

with a fixed $N$ one reaches the noninteracting limit of a homogeneous BEC (depicted with solid red lines in Fig. 5). We need to keep in mind that the atom-photon interaction is proportional to the atom number and not to the density.

In Fig. 6, we show the excitation spectrum above the mean-field along the upper branch solution. Far off the cavity resonance domain, i.e., for $\delta_{C}<-8000 \omega_{R}$ or $\delta_{C}>$ $2000 \omega_{R}$, the spectrum exhibits the excitation levels of a trapped condensate being approximately in the collision dominated Thomas-Fermi regime 25, 26. One feature of the plot is that these levels represent an inert background of almost horizontal lines throughout the detuning range considered. There is a jump in these lines at $\delta_{C}=-6800 \omega_{R}$ which corresponds to the stability threshold of the upper branch. Below this detuning the mean field solution must be on the lower branch of Fig. 5 with less cavity intensity and thus less perturbation of the external trapping potential $V_{\text {ext }}$. The main feature of this plot is that the optomechanical mode appears clearly and its spectral line resembles very much to that of the homogeneous system shown in Fig. 3. This relevant optomechanical mode grows out from the one which has about $4.47 \omega_{R}$ frequency without cavity-BEC interaction, e.g., in the far detuned regime given by the left and right extremes of the plot. Its wave function overlaps the most with the cavity mode function, hence it dominantly couples to the cavity field fluctuations. In the resonant regime $-6800<\delta_{C} / \omega_{R}<2000$ the excitation frequency of this mode crosses the discrete energy levels of the trapped condensate. More precisely, the coupling of the other modes to the cavity field, though negligibly small, is not exactly zero. Therefore what one can see is a series of avoided crossings (at these points the lines associated with modes swap color). Most of the avoided crossings cannot be numerically resolved. At the sides of the plot, some well resolved avoided crossings show that more than one condensate excitation couples considerably to the cavity. Such 

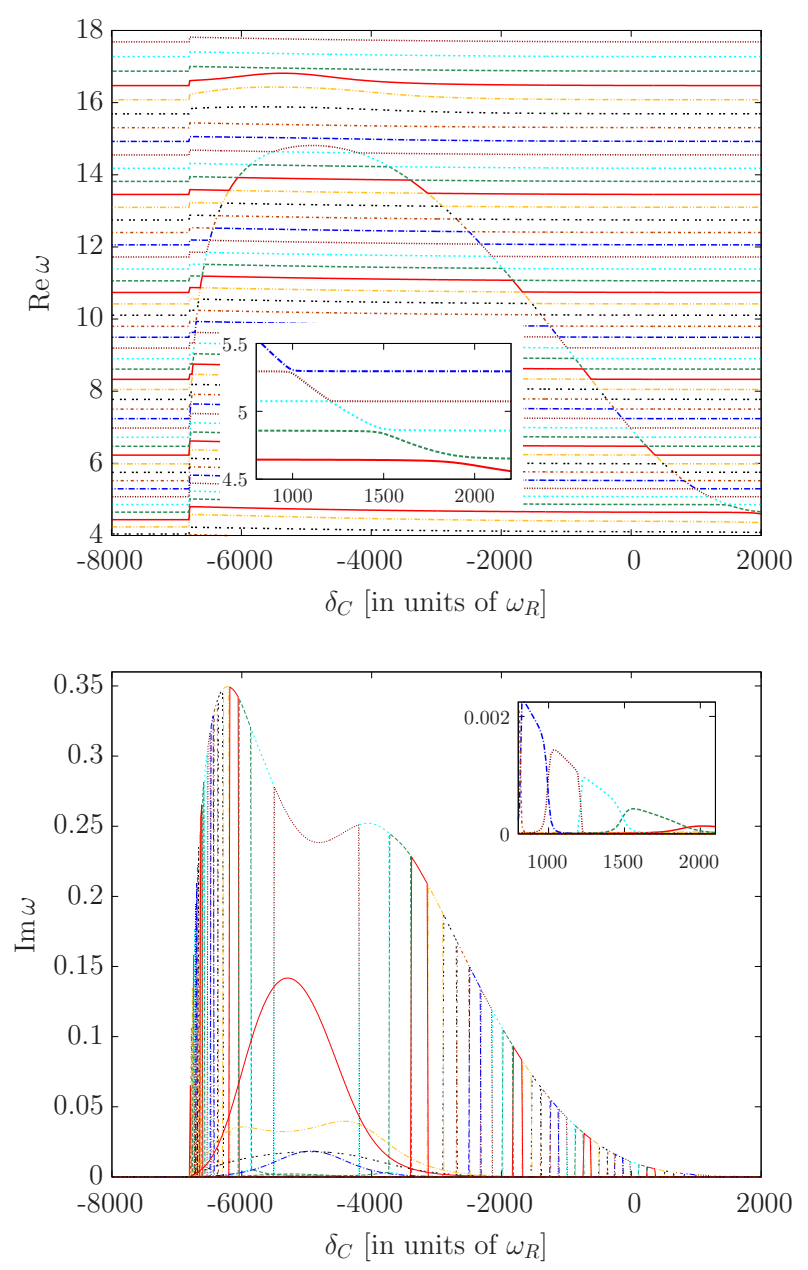

Fig. 6. Frequency (top) and decay rate (bottom) of the relevant optomechanical excitation modes as a function of the cavity detuning $\delta_{C}$ on the upper branch of the bistable regime (cf. with Fig. 3). The insets magnify the avoided crossings for the low lying excitation modes. The plots comprise solely the modes with even parity symmetry. The parameters are: $N=6 \cdot 10^{4}, U_{0}=0.96, \eta=549.5, \kappa=363.9, V_{t r}=0.01$ and $g N=2 \omega_{R}$.

an example is shown in the inset of the Figure at around $\delta_{C} \approx 1500 \omega_{R}$.

The condensate size defines the overlap between the BEC excitation modes and the cavity mode. In Fig. 6 a

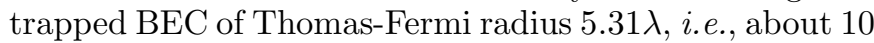
times the optical wavelength leads to a good enough single mode selection and to a behavior similar to that of a homogeneous gas. If the size was reduced, the modes neighboring the optomechanical mode would have larger overlap with the cavity mode. However, their separation in energy would also increase, reducing their effective coupling to the optomechanical dynamics. Reversely, for larger condensate the detuning of the adjacent modes is smaller but the geometrical single mode selection becomes more precise. Finally, note that modes with odd parity do not couple to the cavity field and are not plotted in Fig. 6 at all.
There is an efficient way to identify the condensate excitation modes which couple to the cavity field. These ones have non-vanishing imaginary part in the spectrum (bottom panel of Fig. 6) which is a measure of the coupling. The inset, corresponding to the detuning range selected in the upper panel, clearly demonstrates that two modes are relevant in the narrow vicinity of crossings. Compared to the imaginary part of the spectrum shown in Fig. 3 for homogeneous gas, the envelope indicating the decay rate of the optomechanical mode has a different shape here. In particular, there is a dip at $\delta_{C}=-4800 \omega_{R}$ that can be attributed to the contribution of higher excitation modes. In the homogeneous case, we restricted the Hamiltonian to the lowest two bands, however in the real-space solution all bands are present up to a cutting frequency determined by the discretization. For a homogeneous BEC, the next relevant contribution comes from the $n=2$ band in Eq. (3), since the cavity mode couples the $\cos (2 k x)$ mode to the $\cos (4 k x)$ one. This effect can be clearly recognized here in Fig. 6 in the form of a small modulation of the excitation frequencies at $\delta_{C} \approx-5200 \omega_{R}$ and around the kinetic energy of the $\cos (4 k x)$ mode $\left(16 \omega_{R}\right)$. The mostly affected excitation mode gains a significant decay rate from the cavity interaction (solid red peak at the same detuning).

\section{Conclusion}

A Bose-Einstein condensate, dispersively coupled to the field of a laser-driven high- $Q$ cavity constitutes an alternative system to study cavity optomechanics. Instead of a nanomechanical oscillator, here, a single matter-wave excitation mode couples to the light field, that is selected by the cavity mode function. With this system, a new regime of cavity optomechanics can be studied experimentally.

In this paper, we included s-wave atom-atom collision in the optomechanical model and showed its effects on the optical bistability of the system. For a homogeneous $\mathrm{BEC}$, we derived a radiation pressure Hamiltonian, from which we concluded that collisions increased the frequency of the mechanical oscillator mode and decreased the optomechanical coupling strength. As a result, the bistable regime shrinks.

Due to collisions, a spatially confined gas of degenerate ultracold atoms takes the parabolic profile of the harmonic trap potential. In this case, more than one BEC excitation modes can have finite overlap with the cavity mode function. There is no 'a priori' known excitation mode that would represent a single mechanical oscillator. The calculated excitation spectrum reflects, however, that the single mode picture applies in most of the cases, except for narrow ranges of weak avoided crossings. The contribution of modes around the second harmonics of the cavity mode function was also revealed from the real-space solution.

This work was supported by the Hungarian Academy of Sciences (Lendület Program, LP2011-016) and from the Hungarian National Office for Research and Technology (ERC_HU_09 OPTOMECH). G. Szirmai acknowledges support from the Hungarian National Research Fund (OTKA T077629) and from the János Bolyai Scholarship. 


\section{Appendix}

In the Appendix, we give the explicit form of the matrices appearing in Eqs. (10) and (11). Let us start with the fluctuations of atomic operators with $q \neq 0$. The equations of motion are derived from the Heisenberg equations, $i \hbar \partial_{t} b_{q}=\left[b_{q}, H\right]$ (and similarly for $\left.b_{q}^{\dagger}\right)$, with the Hamiltonian (5), by including the mean-field expansion Eq. (8), and linearizing in the operators. The photon field do not couple to these fluctuations since it carries zero quasimomentum. For the $\mathbf{L}_{q}$ matrix, appearing in the equation of motion 10 , with a straightforward algebra one obtains:

$$
\left[\begin{array}{cccccc}
\omega_{q}^{b} & \mathcal{G} & \mathcal{B} & \mathcal{C} & 0 & 0 \\
-\mathcal{G}-\omega_{q}^{b} & -\mathcal{C} & -\mathcal{B} & 0 & 0 \\
\mathcal{B} & \mathcal{C} & \omega_{q}^{c} & \mathcal{G}\left(1+\frac{\gamma_{0}^{2}}{2}\right) & 4 i q & 0 \\
-\mathcal{C} & -\mathcal{B} & -\mathcal{G}\left(1+\frac{\gamma_{0}^{2}}{2}\right) & -\omega_{q}^{c} & 0 & -4 i q \\
0 & 0 & -4 i q & 0 & \omega_{s}^{q} & \frac{\mathcal{G}}{2}\left(1+\beta_{0}^{2}\right) \\
0 & 0 & 0 & 4 i q & -\frac{\mathcal{G}}{2}\left(1+\beta_{0}^{2}\right) & -\omega_{s}^{q}
\end{array}\right]
$$

with

$$
\begin{aligned}
\omega_{q}^{b} & =\omega_{R} q^{2}+2 \mathcal{G}-\mu, \\
\omega_{q}^{c} & =\omega_{R}\left(4+q^{2}\right)+2 \mathcal{G}-\mu, \\
\mathcal{C} & =2 \mathcal{G} \beta_{0} \gamma_{0}, \\
\mathcal{B} & =u|\alpha|^{2}+2 \mathcal{C} .
\end{aligned}
$$

The equations of motion for the fluctuations of atomic operators with $q=0$ are also obtained directly from their Heisenberg equations and the mean-field substitution (8), but now Eq. (2) is also needed since the photon field couples to these polariton like modes. Finally, the matrix $\mathbf{M}$ of Eq. 11] reads,

$$
\left[\begin{array}{cccccc}
\mathcal{A} & 0 & u \alpha \gamma_{0} & u \alpha \gamma_{0} & u \alpha \beta_{0} & u \alpha \beta_{0} \\
0 & -\mathcal{A}^{*} & -u \alpha^{*} \gamma_{0} & -u \alpha^{*} \gamma_{0} & -u \alpha^{*} \beta_{0} & -u \alpha^{*} \beta_{0} \\
u \alpha^{*} \gamma_{0} & u \alpha \gamma_{0} & \omega_{b} & \mathcal{G} & \mathcal{B} & \mathcal{C} \\
-u \alpha^{*} \gamma_{0}-u \alpha \gamma_{0} & -\mathcal{G} & -\omega_{b} & -\mathcal{C} & -\mathcal{B} \\
u \alpha^{*} \beta_{0} & u \alpha \beta_{0} & \mathcal{B} & \mathcal{C} & \omega_{c} & \mathcal{G}\left(1+\frac{\gamma_{0}^{2}}{2}\right) \\
-u \alpha^{*} \beta_{0}-u \alpha \beta_{0} & -\mathcal{C} & -\mathcal{B} & -\mathcal{G}\left(1+\frac{\gamma_{0}^{2}}{2}\right) & -\omega_{c}
\end{array}\right]
$$

with

$$
\begin{aligned}
\mathcal{A} & =-\delta_{C}+2 \beta_{0} \gamma_{0} N u-i \kappa \\
\omega^{b} & =2 \mathcal{G}-\mu \\
\omega^{c} & =4 \omega_{R}+\mathcal{G}\left(2+\gamma_{0}^{2}\right)-\mu .
\end{aligned}
$$

4. T.P. Purdy, D.W.C. Brooks, T. Botter, N. Brahms, Z.Y. Ma, D.M.S. Kurn, Phys. Rev. Lett. 105, 133602 (2010)

5. D.W.C. Brooks, T. Botter, S. Schreppler, T.P. Purdy, N. Brahms, D.M. Stamper-Kurn, Nature 488, 476 (2012)

6. S. Slama, S. Bux, G. Krenz, C. Zimmermann, P.W. Courteille, Phys. Rev. Lett. 98, 053603 (2007)

7. S. Slama, G. Krenz, S. Bux, C. Zimmermann, P.W. Courteille, Phys. Rev. A 75, 063620 (2007)

8. S.K. Steinke, P. Meystre, Phys. Rev. A 84, 023834 (2011)

9. K. Zhang, P. Meystre, W. Zhang, Phys. Rev. Lett. 108, 240405 (2012)

10. F. Brennecke, T. Donner, S. Ritter, T. Bourdel, M. Köhl, T. Esslinger, Nature 450, 268 (2007)

11. F. Brennecke, S. Ritter, T. Donner, T. Esslinger, Science 322, 235 (2008)

12. S. Gupta, K.L. Moore, K.W. Murch, D.M. Stamper-Kurn, Phys. Rev. Lett. 99, 213601 (2007)

13. S. Ritter, F. Brennecke, K. Baumann, T. Donner, C. Guerlin, T. Esslinger, Appl. Phys. B 95, 213 (2009)

14. P. Rabl, Phys. Rev. Lett. 107, 063601 (2011)

15. A. Nunnenkamp, K. Børkje, S.M. Girvin, Phys. Rev. Lett. 107, $063602(2011)$

16. B. Chen, C. Jiang, J.J. Li, K. Di Zhu, Phys. Rev. A 84, $055802(2011)$

17. S. Yang, M.A. Amri, J. Evers, M.S. Zubairy, Phys. Rev. A 83, 053821 (2011)

18. D. Nagy, P. Domokos, A. Vukics, H. Ritsch, Eur. Phys. J. D 55, 659 (2009)

19. G. Szirmai, D. Nagy, P. Domokos, Phys. Rev. Lett. 102, $080401(2009)$

20. G. Szirmai, D. Nagy, P. Domokos, Phys. Rev. A 81, 043639 (2010)

21. A. Dalafi, M.H. Naderi, M. Soltanolkotabi, Sh, Phys. Rev. A 87, 013417 (2013)

22. P. Domokos, H. Ritsch, J. Opt. Soc. Am. B 20, 1098 (2003)

23. B. Prasanna Venkatesh, J. Larson, D.H.J. O'Dell, Phys. Rev. A 83, 063606 (2011)

24. G. Kónya, G. Szirmai, P. Domokos, Eur. Phys. J. D 65, $33(2011)$

25. A. Csordás, R. Graham, Phys. Rev. A 59, 1477 (1999)

26. C. Menotti, S. Stringari, Phys. Rev. A 66, 043610 (2002)

\section{References}

1. P. Meystre, Ann. Phys. 525, 215 (2012)

2. E. Verhagen, S. Deleglise, S. Weis, A. Schliesser, T.J. Kippenberg, Nature 482, 63 (2012)

3. D.M. Stamper-Kurn, Cavity optomechanics with cold atoms in Cavity optomechanics, edited by M. Aspelmeyer, T. Kippenberg, and F. Marquardt (Springer), arXiv:1204.4351 (2012) 